\title{
ON RADICALS OF INFINITE MATRIX RINGS
}

\author{
by A. D. SANDS \\ (Received 8th March 1968)
}

\section{Introduction}

Let $R$ be a ring and $I$ an infinite set. We denote by $M(R)$ the ring of row finite matrices over $I$ with entries in $R$. The set $I$ will be omitted from the notation, as the same index set will be used throughout the paper. For convenience it will be assumed that the set of natural numbers is a subset of $I$.

Formally $M(R)$ is the set of mappings $m$ from $I \times I$ to $R$ with the restriction that, for each $i$,

$$
I(\boldsymbol{m}, i)=\{j \in I \mid \boldsymbol{m}(i, j) \neq 0\}
$$

is a finite subset of $I$; addition is defined in $M(R)$ pointwise by

$$
(\boldsymbol{m}+\boldsymbol{n})(i, j)=\boldsymbol{m}(i, j)+\boldsymbol{n}(i, j),
$$

and multiplication is defined in $M(R)$ by the usual matrix formula

$$
(\boldsymbol{m n})(i, j)=\sum_{k} \boldsymbol{m}(i, k) \boldsymbol{n}(k, j),
$$

where the summation is taken over the finite set $I(\boldsymbol{m}, i)$, empty summations being interpreted as zero. Then, as is well-known, $M(R)$ forms a ring with respect to these operations.

If $A$ is an ideal of $R$ and $c$ is an infinite cardinal then $M(A, c)$ denotes the set of matrices $m \in M(R)$ such that

(i) $\boldsymbol{m}(i, j) \in A$ for all $i, j \in I$,

(ii) $J(\boldsymbol{m})=\{j \in I \mid \boldsymbol{m}(i, j) \neq 0$, for some $i$, depending on $j\}$ has cardinality less than $c$.

It is easily verified that $M(A, c)$ is an ideal of $M(R)$. If $c$ exceeds the cardinality of $I$ then $M(A, c)=M(A)$. If $c=\aleph_{0}$, the least infinite cardinal, then $M\left(A, \aleph_{0}\right)$ consists of matrices, each with only a finite number of non-zero columns, called by Patterson (4) the ring of row-bounded matrices over $A$.

In $(4,5)$ Patterson has considered the Jacobson radical of $M(R, c)$. He has shown that if $J$ is the Jacobson radical of $R$ then the Jacobson radical of $M\left(R, \aleph_{0}\right)$ is $M\left(J, \aleph_{0}\right)$; but that, for $c>\aleph_{0}$, the Jacobson radical of $M(R, c)$ is $M(J, c)$ if and only if $J$ is right vanishing. In this paper corresponding questions are considered for various other radicals of a ring. These are the p-radical (lower Baer radical), Levitzki radical and nil radical (upper Baer radical). For their definitions and fundamental properties we refer the reader to Divinsky (2). We shall show that for each of these radicals, if $A$ denotes the appropriate radical of $R$, then for $c>N_{0}$ the corresponding radical of 
$M(R, c)$ is $M(A, c)$ if and only if $A$ is nilpotent. For the first two radicals it is shown that the radical of $M\left(R, \aleph_{0}\right)$ is always $M\left(A, \aleph_{0}\right)$. But for the nil radical this problem remains unsolved.

In the last section of the paper the Jacobson radical of $M(R)$, where $R$ is expressed as a direct sum of rings $R_{k}$, is calculated in terms of the Jacobson radicals of $M\left(R_{k}\right)$. The result is then used to give a negative answer to a question raised by Patterson (5).

\section{Ideals of $\mathbf{M}(\mathbf{R})$}

Let $X$ be an ideal of $M(R)$. For each pair $i, j \in I$ let $X(i, j)$ denote the set of elements $\boldsymbol{m}(i, j)$, where $\boldsymbol{m} \in X$. Then it is easily verified that $X(i, j)$ is an ideal of $R$ and that

$$
R X(i, j) \subset X(u, j), \quad X(i, j) R \subset X(i, v), \quad R X(i, j) R \subset X(u, v)
$$

for all $i, j, u, v \in I$.

For $a \in R$ we denote by $a E(i, j)$ the matrix such that $a E(i, j)(i, j)=a$ and $a E(i, j)(u, v)=0$ for $(i, j) \neq(u, v)$. If $a \dot{\in} R a R$ for each $a \in R$, then it may be shown in the usual way (see, for example, Jacobson (3, p. 40)), that if $a \in X(i, j)$ then $a E(u, v) \in X$ and hence that $X(i, j)=X(u, v)$ for all $i, j, u, v \in I$. Then it follows that if $a_{i j}$ is a finite set of elements of $R$, where $(i, j)$ belongs to a finite subset $T$ of $I \times I$, and if $a_{i j} \in X(i, j)$, then the matrix $m=\sum_{T} a_{i j} E(i, j)$ belongs to $X$. In particular these results hold in a ring $R$ with identity element.

Any ring $R$ may be embedded as an ideal in a ring $S$ with identity. In any such embedding if $P$ is a semi-prime ideal of $R$ then $P$ is an ideal of $S$ (see Sands (6)).

If $X$ is an ideal of $M(R)$ then $\bar{X}$ is defined to be the set of matrices $m \in M(R)$ such that $m(i, j) \in X(i, j)$ for all $i, j \in I$. It is clear that $\bar{X}$ is an ideal of $M(R)$ and that $X \subset \bar{X}$. It is not true, in general, that $X=\bar{X}$. For example, it is easily seen that $\overline{M\left(A, \aleph_{0}\right)}=M(A)$.

If $P$ is a prime ideal of $R$ then it may be shown, as in Sands (6), that $M(P, c)$ is a prime ideal of $M(R, c)$. If $P$ is an ideal of $R$ such that $M(P, c)$ is a prime ideal of $M(R, c)$ it may be shown that $P$ is a prime ideal of $R$. But, unlike the case of finite matrices considered in (6), this does not determine all the prime ideals of $M(R, c)$. An example of a primitive ideal, and so a prime ideal, not of this type is given in Sands (7). More directly we show that if $P$ is a prime ideal of $R$ then $M(R, b)+M(P, c)$ is a prime ideal of $M(R, c)$, where $b$ is any infinite cardinal less than $c$.

Let $a, b \notin M(R, b)+M(P, c)$. Then there exist $b$ different columns of $a$ each containing an entry not in $P$. Several of these entries may belong to a given row, but since $a$ is row-finite the number of different rows involved must be $b$. Thus choosing one such entry from each row involved there exists a set $K$ of cardinality $b$ such that $a\left(i_{k}, j_{k}\right) \notin P$ for $k \in K$, where $k_{1} \neq k_{2}$ implies $i_{k_{1}} \neq i_{k_{2}}$ and $j_{k_{1}} \neq j_{k_{2}}$. Similarly there is a set $M$ of cardinality $b$ such that 
$b\left(i_{m}, j_{m}\right) \notin P$ for $m \in M$, where $m_{1} \neq m_{2}$ implies $i_{m_{1}} \neq i_{m_{2}}$ and $j_{m_{1}} \neq j_{m_{2}}$. Let $\alpha$ be a bijection from $K$ to $M$. Since $P$ is a prime ideal of $R$ for each $k \in K$ there exists $r_{k} \in R$ such that $a\left(i_{k}, j_{k}\right) r_{k} b\left(i_{\alpha(k)}, j_{\alpha(k)}\right) \notin P$. Let $r$ be the matrix such that $r\left(j_{k}, i_{\alpha(k)}\right)=r_{k}$, for each $k \in K, r(i, j)=0$ otherwise. Then a routine calculation in matrix multiplication shows that

$$
\operatorname{arb}\left(i_{k}, j_{\alpha(k)}\right)=a\left(i_{k}, j_{k}\right) r_{k} b\left(i_{\alpha(k)}, j_{\alpha(k)}\right) \notin P .
$$

Thus arb contains $b$ different columns, each containing an entry not in $P$. Therefore $a M(R, c) b \not \subset M(R, b)+M(P, c)$. Hence $M(R, b)+M(P, c)$ is a prime ideal of $M(R, c)$.

Theorem 1. If $X$ is a prime ideal of $M(R, c)$ then either $X=M(P, c)$, where $P$ is a prime ideal of $R$, or $X$ contains $M\left(R, \aleph_{0}\right)$.

Proof. If $R$ is embedded as an ideal in an over-ring $S$ with identity then $M(R, c)$ is an ideal of $M(S, c)$ and so $X$ is an ideal of $M(S, c)$. If follows that $X(i, j)=X(u, v)$ for all $i, j, u, v$ in $I$. Let $X(i, j)=P$. Then $P$ is an ideal of $R$. Let $a R b \in P$. Then every element of $a E(1,1) M(R, c) b E(1,1)$ has the form $\operatorname{arb} E(1,1)$ and so is contained in $X$. Thus $a E(1,1) M(R, c) b E(1,1) \subset X$ and $X$ is a prime ideal of $M(R, c)$. Therefore $a E(1,1) \in X$ or $b E(1,1) \in X$. Thus $a \in X(1,1)=P$ or $b \in X(1,1)=P$. It follows that $P$ is a prime ideal of $R$ and that $X \subset M(P, c)$.

Let $\boldsymbol{m}$ be a matrix of $M\left(R, \aleph_{0}\right)$. Let $J=J(\boldsymbol{m})$ be the finite subset of $I$, indexing the non-zero columns of $\boldsymbol{m}$. Let $n$ be a matrix of $M(P, c)$. Let $n^{J}$ be the matrix such that

$$
n^{J}(i, j)=n(i, j), \text { for } i \in J, n^{J}(i, j)=0, \text { otherwise. }
$$

Clearly $\boldsymbol{m n}=\boldsymbol{m} \boldsymbol{n}^{J}$. But $\boldsymbol{n}^{J} \in M(P, c)$ and has only a finite number of non-zero rows and so only a finite number of non-zero entries. Therefore $n^{J} \in X$. It follows that $m \boldsymbol{m} \in X$ and so that $M\left(R, \aleph_{0}\right) M(P, c) \subset X$. Since $X$ is a prime ideal either $M(P, c) \subset X$ or $M\left(R, \aleph_{0}\right) \subset X$. But $X \subset M(P, c)$. Therefore either $M(P, c)=X$ or $M\left(R, \aleph_{0}\right) \subset X$.

Corollary 1. $X$ is a prime ideal of $M\left(R, \aleph_{0}\right)$ if and only if $X=M\left(P, \aleph_{0}\right)$, where $P$ is a prime ideal of $R$.

Corollary 2. $X$ is a primitive ideal of $M\left(R, \aleph_{0}\right)$ if and only if $X=M\left(P, \aleph_{0}\right)$, where $P$ is a primitive ideal of $R$.

This follows from Corollary 1 and from Theorem 3 of Sands (7).

Theorem 2. If $c>\aleph_{0}$ the following conditions are equivalent:

(i) $A$ is a nilpotent ideal of $R$,

(ii) $M(A, c)$ is a nilpotent ideal of $M(R, c)$,

(iii) $M(A, c)$ is a nil ideal of $M(R, c)$.

Proof. $A^{k}=0$ if and only if $M(A, c)^{k}=0$. Therefore conditions (i) and (ii) are equivalent for all infinite cardinals $c$. Clearly (ii) implies (iii) for all infinite cardinals $c$. 
Now, let $c>\aleph_{0}$, and let $A$ be an ideal of $R$, such that $A$ is not nilpotent. Then for each positive integer $k$ there exist elements $a_{k 1}, a_{k 2}, \ldots, a_{k k}$ of $A$ such that $a_{k 1} a_{k 2} \ldots a_{k k} \neq 0$. Let $\boldsymbol{m}$ be the matrix such that $\boldsymbol{m}(i, i+1)=a_{k r}$, where $i$ is a positive integer and $k, r$ are given by $i=1+2+\ldots+(k-1)+r$, $0<r \leqq k$, and $\boldsymbol{m}(u, v)=\mathbf{0}$ otherwise. Since $c>\boldsymbol{N}_{0}$ the matrix $\boldsymbol{m}$ belongs to $M(A, c)$. Then a routine calculation in matrix multiplication shows that, for each positive integer $k, m^{k}(i, i+k)=a_{k 1} a_{k 2} \ldots a_{k k} \neq 0$, where

$$
i=1+2+\ldots+(k-1)+1 \text {. }
$$

Therefore $\boldsymbol{m}^{k} \neq \mathbf{0}$. Since this is true for each positive integer $k$ the matrix $m$ is not nilpotent. Thus $M(A, c)$ is not a nil ideal. It follows that (iii) implies (i).

This completes the proof.

\section{Radicals of $\mathbf{M}(\mathbf{R})$}

Theorem 3. The p-radical of $M\left(R, \aleph_{0}\right)$ is $M\left(A, \aleph_{0}\right)$, where $A$ is the p-radical of $R$.

Proof. The $p$-radical of a ring is the intersection of all prime ideals of the ring. By Corollary 1 to Theorem 1 if $P_{\alpha}$ is the family of all prime ideals of $R$ then, $M\left(P_{\alpha}, \aleph_{0}\right)$ is the family of all prime ideals of $M\left(R, \aleph_{0}\right)$. Hence the $p$ radical of $M\left(R, \aleph_{0}\right)$ is obtained as

$$
\cap M\left(P_{\alpha}, \aleph_{0}\right)=M\left(\cap P_{\alpha}, \aleph_{0}\right)=M\left(A, \aleph_{0}\right) .
$$

In a similar way Corollary 2 to Theorem 1 shows that the Jacobson radical of $M\left(R, \aleph_{0}\right)$ is $M\left(J, \aleph_{0}\right)$ where $J$ is the Jacobson radical of $R$. This result was obtained by Patterson (4) using quasi-regularity of elements.

Theorem 4. If $c>\aleph_{0}$ then the p-radical of $M(R, c)$ is $M(A, c)$, where $A$ is the p-radical of $R$, if and only if $A$ is nilpotent.

Proof. Let $P_{\alpha}$ be the family of prime ideals of $R$. Then $M\left(P_{\alpha}, c\right)$ are prime ideals in $M(R, c)$. Therefore the $p$-radical of $M(R, c)$ is contained in

$$
\cap M\left(P_{\alpha}, c\right)=M(A, c) \text {. }
$$

If $A$ is nilpotent then $M(A, c)$ is nilpotent and so is contained in the $p$ radical of $M(R, c)$. Thus, if $A$ is nilpotent, the $p$-radical of $M(R, c)$ is $M(A, c)$.

If $A$ is not nilpotent then, by Theorem $2, M(A, c)$ is not a nil ideal. But the $p$-radical of a ring is a nil ideal. Therefore $M(A, c)$ cannot be the $p$-radical of $M(R, c)$.

Theorem 5. The Levitzki radical of $M(R, c)$ is zero if and only if the Levitzki radical of $R$ is zero.

Proof. Suppose the Levitzki radical of $R$ is zero. Then if $Y$ is any nonzero ideal of $R, Y$ contains a finitely generated subring which is not nilpotent. Let $X$ be the Levitzki radical of $M(R, c)$. Then $X$ is a semi-prime ideal of $M(R, c)$ and so is an ideal of $M(S, c)$. Since $S$ has an identity, $X(i, j)=X(u, v)$ for all $i, j, u, v \in I$. Let $X(i, j)=W$. Then $W$ is an ideal of $R$. Let $r_{1}, r_{2}, \ldots, r_{n}$ 
be any finite set of elements of $W$. Then $r_{1} E(1,1), \ldots, r_{n} E(1,1)$ belong to $X$ and so generate a nilpotent subring of $M(R, c)$. It follows easily that $r_{1}, \ldots, r_{n}$ general a nilpotent subring of $R$. Therefore $W$ is a locally nilpotent ideal of $R$. Hence $W=0$. But $X \subset M(W, c)$. Therefore $X=0$.

Conversely let $M(R, c)$ have zero Levitzki radical. Let $L$ be the Levitzki radical of $R$. Let $m_{k}, k \in K$, be a finite set of matrices of $M\left(L, \aleph_{0}\right)$. Let $J=\cup J\left(m_{k}\right)$ Then $J$ is a finite subset of $I$. Then the subring of $R$ generated by the finite set of elements of $L, m_{k}(i, j)$, where $k \in K, i, j \in J$, is nilpotent, say of index $t$. Consider the product of $t+1$ matrices $\boldsymbol{m}_{k_{0}}, \boldsymbol{m}_{\boldsymbol{k}_{1}}, \ldots, \boldsymbol{m}_{\boldsymbol{k}_{t}}$, where $k_{i} \in K$. Let $\boldsymbol{m}_{\boldsymbol{k}}^{\boldsymbol{J}}$ be defined by $\boldsymbol{m}_{k}^{J}(i, j)=\boldsymbol{m}_{k}(i, j)$, if $i \in J, \boldsymbol{m}_{k}^{J}(i, j)=0$, otherwise. Then it is easily seen that

$$
\boldsymbol{m}_{k_{0}} \boldsymbol{m}_{\boldsymbol{k}_{1}} \ldots \boldsymbol{m}_{\boldsymbol{k}_{\boldsymbol{t}}}=\boldsymbol{m}_{\boldsymbol{k}_{0}} \boldsymbol{m}_{\boldsymbol{k}_{1}}^{J} \ldots \boldsymbol{m}_{\boldsymbol{k}_{\boldsymbol{t}}}^{J}
$$

But the product of $t$ elements of the form $m_{k}(i, j), i \in J$, is zero. Therefore $m_{k_{1}}^{J} \ldots m_{k_{t}}^{J}=0$. Thus $m_{k_{0}} m_{k_{1}} \ldots m_{k_{t}}=0$. It follows that the finite set of matrices $\boldsymbol{m}_{k}$ generate a nilpotent subring of index at most $t+1$. Therefore $M\left(L, \aleph_{0}\right)$ is a locally nilpotent ideal of $M(R, c)$. But $M(R, c)$ has zero Levitzki radical. Therefore $M\left(L, \aleph_{0}\right)=0$ and so $L=0$.

Corollary. If $P$ is an ideal of $R$ then $R / P$ has zero Levitzki radical if and only if $M(R, c) / M(P, c)$ has zero Levitzki radical.

This follows since $M(R, c) / M(P, c)$ is isomorphic to $M(R / P, c)$.

Theorem 6. The Levitzki radical of $M\left(R, \aleph_{0}\right)$ is $M\left(L, \aleph_{0}\right)$, where $L$ is the Levitzki radical of $R$.

Proof. By a result of Babic (1) the Levitzki radical of a ring $R$ is the intersection of all prime ideals $P_{\alpha}$ of $R$ such that $R / P_{\alpha}$ has zero Levitzki radical. It follows from Corollary 1 to Theorem 1 and the Corollary to Theorem 5 that $M\left(P_{\alpha}, \aleph_{0}\right)$ is then the family of all prime ideals of $M\left(R, \aleph_{0}\right)$ such that $M\left(R, \aleph_{0}\right) / M\left(P_{\alpha}, \aleph_{0}\right)$ has zero Levitzki radical. Therefore the Levitzki radical of $M\left(R, \aleph_{0}\right)$ is obtained as

$$
\cap M\left(P_{a}, \aleph_{0}\right)=M\left(\cap P_{a}, \aleph_{0}\right)=M\left(L, \aleph_{0}\right) \text {. }
$$

Theorem 7. If $c>\aleph_{0}$ then the Levitzki radical of $M(R, c)$ is $M(L, c)$, where $L$ is the Levitzki radical of $R$, if and only if $L$ is nilpotent.

Proof. Let $L=\cap P_{\alpha}$, where $P_{\alpha}$ is the family of all prime ideals of $R$ such that $R / P_{\alpha}$ has zero Levitzki radical. Then, by the Corollary to Theorem 5 , $M(R, c) / M\left(P_{\alpha}, c\right)$ has zero Levitzki radical and $M\left(P_{a}, c\right)$ is a prime ideal of $M(R, c)$. Therefore the Levitzki radical of $M(R, c)$ is contained in

$$
\cap M\left(P_{\alpha}, c\right)=M(L, c) \text {. }
$$

Let $L$ be nilpotent. Then $M(L, c)$ is nilpotent and so $M(L, c)$ is contained in the Levitżki radical of $M(R, c)$. Therefore $M(L, c)$ is the Levitzki radical of $M(R, c)$.

Suppose that $L$ is not nilpotent. Then, by Theorem $2, M(L, c)$ is not a 
nil ideal. But the Levitzki radical of a ring is a nil ideal. Therefore $M(L, c)$ cannot be the Levitzki radical of $M(R, c)$.

Theorem 8. If the nil radical of $R$ is zero then the nil radical of $M(R, c)$ is zero.

Proof. Let $X$ be a nil ideal of $M(R, c)$. Let $x \in X(i, j)$, where $m \in X$ and $\boldsymbol{m}(i, j)=x$. Then $x E(1, i) \boldsymbol{m x} E(j, 1)=x^{3} E(1,1) \in X$. Therefore $x^{3} E(1,1)$ is nilpotent and so $x^{3}$, and hence $x$, is nilpotent. Therefore $X(i, j)$ is a nil ideal of $R$. But $R$ has zero nil radical. Therefore $X(i, j)=0$ for all $i, j \in I$. Hence $X \subset \bar{X}=0$. It follows that $M(R, c)$ has zero nil radical.

We are unable to prove the converse of Theorem 8 and so we do not obtain a precise result for the nil radical of $M\left(R, \aleph_{0}\right)$.

Theorem 9. If $N$ is the nil radical of $R$ then the nil radical of $M(R, c)$ is contained in $M(N, c)$.

Proof. The nil radical of $R$ is the intersection of all prime ideals $P_{\alpha}$ such that $R / P_{\alpha}$ has zero nil radical (see Divinsky (2, pp. 146-147)). It follows that $M(R, c) / M\left(P_{a}, c\right)$ has zero nil radical. Therefore the nil radical of $M(R, c)$ is contained in $\cap M\left(P_{\alpha}, c\right)=M(N, c)$.

Theorem 10. If $c>\aleph_{0}$ then the nil radical of $M(R, c)$ is $M(N, c)$, where $N$ is the nil radical of $R$, if and only if $N$ is nilpotent.

Proof. If $N$ is nilpotent then $M(N, c)$ is nilpotent and so is contained in the nil radical of $M(R, c)$. It follows from Theorem 9 that $M(N, c)$ is the nil radical of $M(R, c)$.

If $N$ is not nilpotent then, by Theorem 2, $M(N, c)$ is not a nil ideal. But the nil radical of a ring is a nil ideal. Hence $M(N, c)$ cannot be the nil radical of $M(R, c)$.

The failure to obtain a precise result for the nil radical of $M\left(R, \aleph_{0}\right)$ is not due to any weakness in the techniques for handling infinite matrices but reflects the lack of knowledge about the nil radical of finite matrix rings. Let $M(R, n)$ denote the ring of $n \times n$ matrices with entries in $R$, where $n$ is a positive integer. If for each $n$ the nil radical of $M(R, n)$ is $M(N, n)$, where $N$ is the nil radical of $R$, then the nil radical of $M\left(R, \aleph_{0}\right)$ is $M\left(N, \aleph_{0}\right)$. For if $m$ is a matrix of $M\left(N, \aleph_{0}\right)$ and $m^{J(m)}$ is defined as before then $m^{J(m)}$ is essentially an $n \times n$ matrix, bordered with zeros, where $n$ is the cardinality of the finite set $J(m)$. The $n \times n$ matrix belongs to $M(N, n)$, which we have assumed to be a nil ideal. Therefore there exists an integer $k$ such that $\left(m^{J(m)}\right)^{k}=0$. Then

$$
m^{k+1}=m\left(m^{J(m)}\right)^{k}=0 .
$$

Hence $M\left(N, \aleph_{0}\right)$ is a nil ideal. It follows from Theorem 9 that $M\left(N, \aleph_{0}\right)$ is the nil radical of $M\left(R, \aleph_{0}\right)$, as required. Conversely if $M\left(N, \aleph_{0}\right)$ is the nil radical of $M\left(R, \aleph_{0}\right)$ then any matrix of $M(N, n)$ may be bordered with zeros and regarded as a matrix of $M\left(N, \aleph_{0}\right)$. Therefore it is nilpotent and so $M(N, n)$ is a nil ideal. By the analogue of Theorem 9 for finite matrices, $M(N, n)$ is 
the nil radical of $M(R, n)$. Thus the problem of whether the nil radical of $M\left(R, \aleph_{0}\right)$ is $M\left(N, \aleph_{0}\right)$ is equivalent to the same problem for all the finite matrix rings $M(R, n)$.

\section{The Jacobson radical of a matrix ring over a direct sum of rings}

Let $K$ be an infinite set, such that with each $k \in K$ there is associated a ring $R_{k}$. Let $R=\oplus R_{k}$ be the (restricted) direct sum of the rings $R_{k}$, i.e. if $\theta \in R$ and $\theta(k) \in R_{k}$ is the $k$ th component of $\theta$, then

$$
K(\theta)=\{k \in K \mid \theta(k) \neq 0\}
$$

is a finite subset of $K$. For each $k \in K$, we denote the canonical injection from $R_{k}$ to $R$ by $\eta_{k}$, i.e. for $x \in R_{k}, \eta_{k}(x)$ is defined by $\eta_{k}(x)(k)=x, \eta_{k}(x)(m)=0$, $m \neq k$. The canonical projection from $R$ to $R_{k}$ is denoted by $\pi_{k}$, i.e. for $\theta \in R, \pi_{k}(\theta)=\theta(k)$.

If $M(R, c)$ and $M\left(R_{k}, c\right)$ are matrix rings over $R$ and $R_{k}$ then $\eta_{k}$ and $\pi_{k}$ induce homomorphisms from $M\left(R_{k}, c\right)$ to $M(R, c)$ and from $M(R, c)$ to $M\left(R_{k}, c\right)$. We shall denote these homomorphisms by $\eta_{k}$ and $\pi_{k}$, though strictly speaking they should involve $M$ and $c$. Thus $\eta_{k}$ from $M\left(R_{k}, c\right)$ to $M(R, c)$ is defined by $\left(\eta_{k}(m)\right)(i, j)=\eta_{k}(m(i, j))$, for each $m \in M\left(R_{k}, c\right)$, and $\pi_{k}$ from $M(R, c)$ to $M\left(R_{k}, c\right)$ is defined by $\left(\pi_{k}(\theta)\right)(i, j)=\pi_{k}(\theta(i, j))$ for each $\theta \in M(R, c)$. It should be noted that the processes of forming infinite matrix rings and infinite direct sums do not commute. $\sum_{k} \eta_{k} M\left(R_{k}, c\right)$ is an ideal of $M(R, c)$. But it is a proper ideal, since if $\theta \in \sum_{k} \eta_{k} M\left(R_{k}, c\right)$ then there is a finite subset $K_{0}$ of $K$ such that $\pi_{k} \theta(i, j)=0$ for $k \notin K_{0}$ and all $i, j \in I$. Whereas for a general $\theta$ in $M(R, c)$ there is, for each $i, j$, a finite subset $K(\theta(i, j))$ such that $\pi_{k} \theta(i, j)=0$ for $k \notin K(\theta(i, j))$, but the union of these finite subsets $K(\theta(i, j))$, taken over all $(i, j) \in I \times I$, need not be finite.

Theorem 11. If $R=\oplus R_{k}$ and $J_{k}$ is the Jacobson radical of $M\left(R_{k}, c\right)$, then the Jacobson radical of $M(R, c)$ is the set $A$ of matrices $\theta \in M(R, c)$ such that $\pi_{k}(\theta) \in J_{k}$, for all $k \in K$.

Proof. Let $\theta$ belong to the Jacobson radical of $M(R, c)$. Then $\pi_{k}(\theta)$ belongs to the Jacobson radical of $\pi_{k}(M(R, c))=M\left(R_{k}, c\right)$ for all $k \in K$. Therefore $A$ contains the Jacobson radical of $M(R, c)$.

Let $\theta \in A$. Let $\pi_{k}(\theta)=\theta_{k}$. Then $\theta_{k} \in J_{k}$, which is a quasi-regular ideal of $M\left(R_{k}, c\right)$. Therefore there exists $\phi_{k} \in J_{k}$ such that $\theta_{k}+\phi_{k}-\theta_{k} \phi_{k}=0$. It follows from this relation that if row $i$ of $\theta_{k}$ is zero then row $i$ of $\phi_{k}$ is zero. Since $\theta$ is row-finite, for each $i \in I$ there is only a finite number of indices $j \in I$ such that $\theta(i, j) \neq 0$. Corresponding to each such $\theta(i, j)$ there is only a finite number of indices $k \in K$ such that $\theta(i, j)(k) \neq 0$. Hence if we write

$$
K_{i}=\left\{k \in K \mid \text { row } i \text { of } \theta_{k} \text { is non-zero }\right\}
$$

it is clear that $K_{i}$ is a finite subset of $K$. For each $j \in I$ we define $\phi(i, j)$ by $\phi(i, j)(k)=\phi_{k}(i, j)$ for all $k \in K$. Then $K(\phi(i, j))=\{k \in K \mid \phi(i, j)(k) \neq 0\}$ is E.M.S. $-\mathrm{N}$ 
contained in $K_{i}$, which is finite. Therefore $\phi(i, j) \in R$. Since $\phi_{k}$ is row-finite for all $k$ and row $i$ of $\phi_{k}$ is zero for $k \notin K_{i}$, where $K_{i}$ is finite, it follows that the matrix $\phi$ is row-finite. Hence $\phi \in M(R, d)$, for some infinite cardinal $d$. Then

$$
\begin{aligned}
\left(\pi_{k}(\theta+\phi-\theta \phi)\right)(i, j) & =\pi_{k}(\theta(i, j)+\phi(i, j)-(\theta \phi)(i, j)) \\
& =\theta_{k}(i, j)+\phi_{k}(i, j)-\left(\theta_{k} \phi_{k}\right)(i, j)=\left(\theta_{k}+\phi_{k}-\theta_{k} \phi_{k}\right)(i, j)=0 .
\end{aligned}
$$

Since this is true for all $k \in K$ it follows that $(\theta+\phi-\theta \phi)(i, j)=0$ for all $i, j \in I$. Hence $\theta+\phi-\theta \phi=0$. Then $\phi=\theta \phi-\theta \in M(R, c)$, since $\theta \in M(R, c)$. Therefore $\theta$ is quasi-regular. It follows that $A$ is a quasi-regular ideal of $M(R, c)$ and so $A$ is contained in the Jacobson radical of $M(R, c)$.

Therefore the Jacobson radical of $M(R, c)$ is $A$.

One object of proving this theorem is to answer in the negative a question of Patterson (5). It is known that, if $J$ is the Jacobson radical of a ring $R$ and $S_{\alpha}$ is the family of all right vanishing ideals of $R$, then $M\left(J, \kappa_{0}\right)+\Sigma M\left(S_{\alpha}, c\right)$ is contained in the Jacobson radical of $M(R, c)$. Patterson (5) asks whether or not this ideal is always equal to the Jacobson radical of $M(R, c)$. We shall now use Theorem 11 to show that equality need not hold.

Let $K$ be the set of natural numbers. For each $k \in K$ let the ring $R_{k}$ be equal to the ring $P$ of $p$-adic integers. It is known that the Jacobson radical of $P$ is $p P$. If $R=\oplus R_{k}$, then $R$ contains no non-zero right vanishing ideal. Let $c>\aleph_{0}$. Then we need only show that the Jacobson radical of $M(R, c)$ is not contained in $M\left(J, \aleph_{0}\right)$, where $J$, the Jacobson radical of $R$, is the direct sum of the Jacobson radicals of $R_{k}$. Let $J_{k}$ be the Jacobson radical of $M\left(R_{k}, c\right)$; then $J_{k}$ contains $M\left(p P, \aleph_{0}\right)$.

Let the matrix $\theta$ be defined by $\theta(i, j)=0, i \neq j, \theta(i, j)=0, i \notin K$,

$$
\pi_{m} \theta(k, k)=0, k, m \in K, k \neq m, \pi_{k} \theta(k, k)=p,
$$

for each $k \in K$. Then $\pi_{k}(\theta)=p E(k, k) \in M\left(p P, \aleph_{0}\right) \subset J_{k}$, for each $k \in K$. Further $\theta \in M(R, c)$, since $c>\aleph_{0}$. Therefore, by Theorem 11, $\theta$ belongs to the Jacobson radical of $M(R, c)$. But $\theta$ does not belong to $M\left(R, \aleph_{0}\right)$ and so $\theta$ does not belong to $M\left(J, \aleph_{0}\right)$. Thus the Jacobson radical of $M(R, c)$ is not equal to $M\left(J, \aleph_{0}\right)+\Sigma M\left(S_{a}, c\right)$.

It should be noted that the analogue of Theorem 11 does not hold for any of the three radicals discussed in Section 3. Let $K$ be the set of natural numbers. Let $R_{k}$ be a nilpotent ring with index of nilpotency $k$, say the ring of even integers modulo $2^{k}$. Let $R$ be the direct sum of the rings $R_{k}$. Then $R$ is a direct sum of nilpotent rings and so is its own radical for each of the three types of radical under discussion. Clearly $R$ is not a nilpotent ring. Hence by Theorems 4,7 and $10, M(R, c)$ is not the appropriate radical of $M(R, c)$, for $c>\aleph_{0}$. But $\pi_{k}(M(R, c))=M\left(R_{k}, c\right)$ and is a nilpotent ring. So by these theorems $M\left(R_{k}, c\right)$ is its own radical of the appropriate type. Hence the ideal $A$ as defined in Theorem 11, in this case, is equal to $M(R, c)$. But we have seen that this is not the radical of $M(R, c)$, where $c>\aleph_{0}$, in any of these three cases. 
Thus we have justified the claim that the analogue of Theorem 11 does not hold.

\section{REFERENCES}

(1) A. M. BABIC, The Levitzki radical, Dokl. Akad. Nauk. SSSR, 126, no. 2 (1959), 242-243.

(2) N. J. DrVINSKY, Rings and Radicals (Toronto, 1965).

(3) N. JaCobson, Structure of Rings (Amer. Math. Soc. Colloq. Publ. 37, 1956).

(4) E. M. PATterson, On the radicals of certain rings of infinite matrices, Proc. Royal Soc. Edinburgh, 65 (1961), 263-271.

(5) E. M. Patterson, On the radicals of rings of row-finite matrices, Proc. Royal Soc. Edinburgh, 66 (1962), 42-46.

(6) A. D. Sands, Prime ideals in matrix rings, Proc. Glasgow Math. Assoc. 2 (1956), 193-195.

(7) A. D. Sands, Primitive rings of infinite matrices, Proc. Edinburgh Math. Soc. (2) 14 (1964), 47-53.

Department of Mathematics

THE UNIVERSITY

DUNDEE 